Erratum

\title{
Nonlinear elliptic equations on compact riemannian manifolds and asymptotics of Emden equations
}

\author{
Marie-Françoise Bidaut-Veron and Laurent Veron \\ Mathématiques, Université de Tours, F-37200 Tours, France \\ Invent. math. 106, 489-539 (1991)
}

S. Ilias pointed us out that in the statement of Theorem 6.1, the equality $(M, g)$ $=\left(S^{n}, g_{0}\right)$ must be understood in the sense of conformally diffeomorphic, that is $(M, g)$ is isometric to $\left(S^{n}, k g_{0}\right)$ for some $k$ positive and $C^{\infty}$ on $S^{n}$.

The same remark holds in the proof on p. 528, which is clear from

$$
\int_{M} v^{\nu}\left(|\operatorname{Hess} v|^{2}-\frac{1}{n}\left(\Delta_{g} v\right)^{2}\right)>0 \text {. }
$$

